\title{
Lidil
}

Revue de linguistique et de didactique des langues

$54 \mid 2016$

La phrase en production d'écrits, approches nouvelles en didactique

\section{Le verbe et la phrase dans des définitions d'élèves : entre production et conceptualisation - ce qu'ils font et ce qu'ils « disent »}

How Pupils Use the Notion of Sentence in Their Definitions of the Verb-What

They Say and What They Do

Marie-Laure Elalouf, Patrice Gourdet et Danièle Cogis

\section{(2) OpenEdition}

Journals

Édition électronique

URL : http://journals.openedition.org/lidil/4047

DOI : $10.4000 /$ lidil.4047

ISSN : 1960-6052

Éditeur

UGA Éditions/Université Grenoble Alpes

Édition imprimée

Date de publication : 20 novembre 2016

Pagination : 55-74

ISBN : 978-2-84310-339-1

ISSN : $1146-6480$

Référence électronique

Marie-Laure Elalouf, Patrice Gourdet et Danièle Cogis, « Le verbe et la phrase dans des définitions d'élèves : entre production et conceptualisation - ce qu'ils font et ce qu'ils « disent » », Lidil [En ligne], 54 | 2016, mis en ligne le 01 janvier 2017, consulté le 10 décembre 2020. URL : http:// journals.openedition.org/lidil/4047 ; DOI : https://doi.org/10.4000/lidil.4047 


\title{
Le verbe et la phrase dans des définitions d'élèves : entre production et conceptualisation - ce qu'ils font et ce qu'ils "disent "
}

\author{
Marie-Laure Elalouf, Patrice Gourdet* \\ et Danièle Cogis**
}

\begin{abstract}
RÉSUMÉ
Sollicités pour répondre à la question «Qu'est-ce qu'un verbe?», 491 élèves de $\mathrm{CM} 2$ ( $5^{\mathrm{e}}$ primaire) répondent en produisant des phrases mais seulement 199 d'entre eux convoquent la notion de phrase dans la définition du verbe. Ce décalage entre activité linguistique et métalinguistique est connu. Nous souhaitons dans cet article le documenter à deux niveaux.
\end{abstract}

Le premier concerne l'appropriation par des élèves de fin de l'école primaire du genre textuel convoqué régulièrement dans le cadre scolaire : la définition d'une notion grammaticale. Elle s'accompagne d'une segmentation où tendent à coïncider phrase syntaxique et phrase graphique. La comparaison avec un corpus composé des mêmes élèves en CE2 trois ans auparavant qui ont répondu à la même question mettra en évidence l'évolution des réponses. Nous étudierons les constructions récurrentes et ferons des hypothèses sur les constructions correspondant à des stratégies de restitution de connaissances.

Le second niveau d'analyse concerne la conceptualisation des notions de verbe et de phrase. Nous dressons une typologie des relations que les élèves établissent entre elles en dégageant celles qui sont prototypiques. Nous nous interrogerons sur ce qu'elles permettent d'inférer concernant la compréhension de la notion de prédication, qui n'était pas enseignée en France lorsque les données ont été collectées.

\footnotetext{
* Université de Cergy-Pontoise, laboratoire EMA, ESPE.

** MoDyCo, UMR 7114 CNRS.
} 


\begin{abstract}
A question was asked of 491 pupils (5th form of primary school): "What's a verb?" They all answered and produced sentences but only 199 of them introduced the notion of sentence in the definition of the verb. This discrepancy between linguistic and metalinguistic activity is known. Our purpose is to study this issue at two levels.

The first one concerns how pupils appropriate a kind of text which is regularly used at school: the definition of a grammatical notion in which syntactic segmentation is provided by punctuation. We compared our results to these obtained in the same pupils three years before. We study the most frequent constructions resulting from the delivery of the knowledge.

The second level of analysis deals with the conceptualisation of the notions of verb and sentence. We establish a classification of the relationships pupils build between these two notions. We show that it is possible to infer that they have an intuitive approach of the notion of predication, though it was not taught in France, when the data were collected.
\end{abstract}

\title{
1. Introduction
}

Lorsqu'ils arrivent à la fin de l'école primaire, les élèves français ont reçu pendant cinq ans un enseignement grammatical dans lequel la notion de phrase est centrale. Première notion introduite au moment de l'apprentissage de la lecture, régulièrement reprise ensuite, non seulement elle est le cadre de la description grammaticale enseignée à l'école primaire (Boré \& Bosredon, 2013, p. 13), mais elle est aussi censée rendre compte de l'organisation syntaxique des règles données à apprendre. C'est pourquoi les productions d'élèves de CM2 (5 primaire), en réponse à la question «Qu'est-ce qu'un verbe?» nous sont apparues comme un poste d'observation pertinent pour accéder aux représentations qu'ils ont élaborées dans le cadre des pratiques discursives ordinaires de la classe (Fisher, 2004).

Cette recherche prolonge un premier travail de recueil et d'analyse auprès des mêmes élèves, à qui la même question avait été posée deux ans plus tôt (Gourdet \& Cogis, 2014, p. 50) ${ }^{1}$. Face à cette sollicitation

1. Le protocole a été proposé à en octobre 2010 à des élèves de CE2 (3 $3^{\mathrm{e}}$ primaire) puis en juin 2013 aux mêmes élèves quand ils étaient en CM2 (5 primaire). Ces 491 élèves sont issus de 32 classes au sein de 15 écoles primaires de la même circonscription. 
appelant la rédaction d'une définition, 491 élèves de CM2 répondent en produisant des séquences phrastiques qui demanderont à être décrites, mais seulement 199 d'entre eux convoquent la notion de phrase dans la définition du verbe. Mais s'agit-il de la même notion?

Ce décalage entre activité linguistique et métalinguistique est connu. Nous souhaitons montrer que des élèves qui lisent et apprennent des définitions intègrent progressivement certaines des caractéristiques syntaxiques de ces phrases sans qu'elles fassent toutes l'objet d'un enseignement explicite et que, parallèlement, ces mêmes élèves conceptualisent les relations entre le verbe et la phrase.

\section{La phrase dans les définitions d'élèves : analyse de quelques caractéristiques en liaison avec le genre textuel de la définition}

Le genre scolaire de la définition est présent dans les règles qui institutionnalisent le savoir enseigné, où prédomine l'énoncé de propriétés suivi d'exemples (Gourdet, 2013, p. 270). Il présente des caractéristiques qui contraignent la construction des phrases. L'une d'elle fait l'objet d'une injonction répétée - l'élève doit commencer sa réponse par une phrase complète - les autres ne peuvent guère être déduites que de la fréquentation de définitions. Nous étudierons d'abord les évolutions majeures qui interviennent dans la construction des phrases entre le début du CE2 et la fin du CM2, puis nous montrerons comment les contraintes syntaxiques du genre textuel influent ou non sur les productions d'élèves. L'enjeu est bien de comprendre la relation entre l'appropriation de ce genre particulier par les élèves et la production de phrases en situation d'écriture autonome.

\subsection{Les évolutions majeures entre le CE2 et le $\mathrm{CM}_{2}$}

La demande de «restitution de savoir» est une pratique répandue à l'école, mais plutôt sous une modalité orale et collective. On peut donc s'attendre à ce que des constructions usuelles dans l'interaction orale se rencontrent dans les productions écrites, en concurrence avec des formulations plus proches de celles des grammaires scolaires et des cahiers de règles qui s'en inspirent. À la question «Qu'est-ce qu'un verbe?», la réponse scolaire attendue est une phrase attributive dont le sujet est «un verbe», mais certains élèves répondent par un énoncé rhématique, dépendant, pour l'interprétation du thème, de la question posée : 
[8] C'est un mot qui est devant un pronom personelle qui se conjugue a plusieur temps. Pour les reconnaître on peut changer le temps de la phrase.

La proportion d'élèves produisant un énoncé syntaxiquement autonome, reprenant un verbe en le thématisant, croit du CE2 au CM2.

\begin{tabular}{|l|c|c|}
\hline & CE2 & CM2 \\
\hline Sans thématisation explicite & $25 \%$ & $16 \%$ \\
\hline Avec thématisation linguistique & $75 \%$ & $84 \%$ \\
\hline
\end{tabular}

Tableau 1. - Répartition des réponses des élèves de $\mathrm{CE}_{2}$ et de $\mathrm{CM}_{2}$ selon la présence ou non d'une thématisation.

Les réponses non autonomes qui subsistent en fin d'école primaire appellent une attention particulière. Leur recueil a exigé une dictée à l'adulte (1) ou requiert un effort d'interprétation, soit que l'élève n'emploie pas de métatermes (2) ou, cas le plus fréquent, fait des erreurs sur les homophones et la segmentation (3):

(1) C'est un mot, il peut se conjuguer (dictée à l'adulte). [484]

(2) C'est ce que l'on fait. C'est aussi quand on a et quand on est ${ }^{2}$. [133]

(3) Ce quon mais dans une phrase pour que on comprène mieux la phrase si non s'ai assez dur à comprendre et les verbes on peut aussi le conjuguer à tout les temps. [121]

Ces réponses sont généralement courtes, décrivant une propriété, ou deux dans des propositions coordonnées, et dépourvues d'exemples. On rencontre plusieurs cas d'absence de majuscule et surtout de ponctuation finale. Pour ces élèves, les compétences en littéracie se révèlent fragiles.

Mais une majorité d'élèves applique la règle qui veut qu'une réponse scolaire soit syntaxiquement autonome. Ils peuvent produire une phrase segmentée ou non. La structure des phrases segmentées produites est celle que Bally (1909) a décrite comme phrases de type AZ, A désignant le thème, détaché en position frontale, $\mathrm{Z}$ le propos ${ }^{3}$. Quant

2. Les graphies proposées par les élèves ont été respectées.

3. Chez Bally, la désignation des éléments $\mathrm{A}$ et $\mathrm{Z}$ varie entre la $1^{\text {re édition de }}$ Linguistique générale et linguistique française (1932) et la seconde (1944) : thème de l'énonciation/énoncé proprement dit; puis thème de l'énoncé/ propos, but de l'énonciation. 
aux phrases non segmentées ou liées, elles correspondent au modèle de la phrase de base (Tomassone, 2002).

\begin{tabular}{|l|c|c|}
\hline & 352 élèves de CE2 & 441 élèves de CM2 \\
\hline $\begin{array}{l}\text { Phrase segmentée } \\
\text { Ex. [59] Un verbe on peut le } \\
\text { conjuguer }\end{array}$ & $41 \%$ & $38 \%$ \\
\hline $\begin{array}{l}\text { Phrase liée } \\
\text { Ex. [6] Un verbe est un mot qui } \\
\text { change le temps }\end{array}$ & $59 \%$ & $62 \%$ \\
\hline
\end{tabular}

Tableau 2. - Répartition des réponses des élèves de CE2 et de $\mathrm{CM}_{2}$ selon la segmentation ou non des phrases produites.

Certaines réponses de CM2 ont les caractéristiques syntaxiques de la langue parlée :

[59] Un verbe on peut le conjuguer il est toujours après le sujet et quand on le conjugue sa terminaison change, mais Attention il y a des terminaison différente il y a au : $1^{\text {er }}$ groupe, $2^{\text {ème }}$ groupe, et au $3^{\text {ème }}$ groupe le verbe on le reconnaît très vite car il est toujours apres le sujet pour les verbes du $\underline{1}^{\mathrm{er}}$ groupe : l'infinitif est : er, au $\underline{2}^{\text {ème }}$ groupe : l'infinitif est : ir, au $3^{\text {ème }}$ groupe : l'infinitif est : oir, dre, tre)

Ici, la syntaxe est celle de l'oral, avec deux phrases segmentées, une rupture syntaxique (il y a au $1^{\text {er }}$ groupe, $2^{\text {ème }}$ groupe et $3^{\text {ème }}$ groupe), une injonction nominale (mais Attention), les accents d'insistance étant matérialisés par des soulignements.

La construction segmentée la plus fréquente en CM2 est celle dont l'attaque est «Un verbe c'est un mot», où le pronom démonstratif reprenant un verbe permet d'introduire le terme générique de la définition, mot (61 occurrences). Cette construction est concurrencée par celle dont l'attaque est «Un verbe est un mot» (128 occurrences), dont on peut faire l'hypothèse qu'elle figure dans les cahiers de règles des élèves ${ }^{4}$.

Cependant, entre le CE2 et le CM2, le rapport entre phrases segmentées et phrases liées évolue peu. On peut s'interroger sur la conscience que les élèves ont de la différence entre les deux constructions, sachant que seule la phrase liée est décrite dans les grammaires scolaires et

4. En France, l'usage du cahier de règles est général, la demande institutionnelle étant renforcée par la pression parentale. 
fait l'objet d'un enseignement. Ainsi, dans les productions écrites des élèves, il est très rare que le thème soit séparé par une virgule (2 occurrences de un verbe, c'est un mot, une de un verbe, c'est quelque chose).

Entre le CE2 et le CM2, les réponses uniquement rhématiques qui relèvent plutôt d'un genre conversationnel ont diminué et celles qui reprennent de la consigne le syntagme un verbe, pour le placer en position de thème, sont majoritaires. Elles s'apparentent au genre de la définition, mais la préférence accordée à la phrase liée dans les définitions écrites ne s'impose pas nettement.

\subsection{Les contraintes syntaxiques du genre définitionnel}

En posant la question «Qu'est-ce qu'un verbe?», l'enquêteur demandait de définir un exemplaire jugé représentatif de la classe entière et attendait la production d'énoncés génériques. C'est le cas de toutes les réponses. Même les deux réponses qui relèvent plus du texte injonctif que du genre définitionnel comportent des expressions génériques et non spécifiques. La question posée appelle une construction phrastique privilégiée : une phrase attributive dont le prédicat comporte un hyperonyme qui informe sur le mode de catégorisation de la notion étudiée et une relative qui introduit une caractéristique. Ce schéma de construction est très bien représenté dans notre corpus. Il concerne aussi bien les phrases autonomes que non autonomes, segmentées que non segmentées :

[6] Un verbe est un mot qui change le temps et il ce conjugne (réponse autonome, phrase liée).

[106] Un verbe c'est un mot qu'on peut conjuguer. Et ça représente une action. Un verbe on peut le conjuguer à plusieurs temps au présent, imparfait, futur, passé simple, passé composé, futur antérieur, au plus que parfait (réponse autonome, phrase segmentée).

[82] Cet un mot qui change en fonction de la phrase. [...] (réponse non autonome).

[21] Quelle que chose qui exprime une manière ou une question. [...] (réponse non autonome).

Une variante consiste à caractériser l'hyperonyme par un adjectif ou un participe fonctionnant comme un adjectif :

[175] Un verbe c'est un mot banale dans une phrase il est toujours accompagné de son sujet. Rien d'autre à dire. 
[123] Un verbe est un mot conjugué et aussi entouré de négation on peut le conjugué avec «je, tu, il, nous, vous, ils» et il peut être entre ne et pas.

Au-delà de cette phrase d'attaque, les contraintes syntaxiques qu'ont à gérer les élèves sont d'ordre transphrastique : comment reprendre une expression générique par une autre expression générique ou un pronom? S'agissant des expressions nominales, ils emploient toute la gamme des possibles : le déterminant défini singulier et le défini pluriel. Le groupe nominal défini singulier se rencontre en concurrence avec les pronoms ça et il.

[62] Un verbe est une action. Quand le verbe est à l'infinitif il se termine par er au premier groupe ir au deuxième groupe et re au troisième groupe.

[32] Un verbe peut être conjugai ou a l'infinitif il peut avoir un sujet il est accompagné d'un pronom. Il est dans une phrase.

[91] Un verbe est un mot conjuguer à plusieur temps et sa s'écrie de diférente magnère.

La reprise anaphorique par ça qui signale sans ambigüité l'interprétation générique, est concurrencée par l'emploi du pronom personnel, jugé plus conforme à la langue écrite, mais qui rend possibles des glissements moins contrôlés du générique au spécifique (voir par exemple [8] en 2.1).

L'emploi du défini pluriel est nécessaire dans la rédaction d'une définition disjonctive, certaines propriétés du verbe étant valables pour la classe entière et d'autres pour une sous-classe seulement. Passer de la définition du verbe au classement des verbes en groupes suppose de passer du singulier au pluriel, ce que font quelques élèves dont les formulations sont très proches des formulations des grammaires :

[37] Un verbe est un mot qui peut se conjuguer à plusieur temps. Les verbes se classe en trois groupe (le $1^{\text {er }}$, le $2^{\text {ème }}$, le $\left.3^{\text {ème}}\right)$. Ils ont des terminaisons différente selon leurs groupe et le temps auquelle on les conjugues.

Mais pour d'autres, la rigidité des patrons lexico-syntaxiques ne permet pas ces variations, comme la répétition du verbe avoir dans l'exemple suivant :

[14] Un verbe a un sujet, il a aussi 3 groupe, $1^{\text {er }}, 2^{\circ}$, et 3 groupe [... 
Par ailleurs, l'emploi du déterminant défini pluriel révèle des problèmes de catégorisation :

[271] Il existe plusieurs sorte de verbe. il y a les verbes conjugués, les verbes a l'infinitif, les verbes du $1^{\text {er }}$ groupe, $2^{\text {ème }}$ groupe et $3^{\text {ème }}$ groupe.

[36] Dans les verbes il y a 3 groupes, le premier, le deuxième et le troisième il y à aussi les auxilière.

L'énumération d'expressions nominales déterminées par les laisse penser que les sortes de verbes se juxtaposent alors que les classements relèvent de niveaux différents et peuvent se combiner.

En dehors de ces difficultés, l'étude de l'ensemble des réponses montre que les élèves usent des ressources de la langue pour produire des énoncés définitoires. Mais c'est sur le plan de la segmentation, marquée ou non par un signe de ponctuation, qu'ils s'écartent néanmoins le plus du genre définitionnel représenté par les cahiers de règles et les manuels.

\subsection{La ponctuation des définitions d'élèves}

Le genre scolaire de la définition grammaticale se caractérise par une segmentation où tendent à coïncider phrase syntaxique et phrase graphique, tant dans les énoncés métalinguistiques que dans les exemples. Dans le corpus de manuels étudié ${ }^{5}$, cette coïncidence est régulière, tandis que dans les réponses des élèves, on compte souvent une phrase graphique pour plusieurs phrases syntaxiques (Paolacci \& Rossi-Gensane, 2012). Les réponses sont des périodes (Berrendonner, 2002) au sens où elles forment un "programme discursif complet», clos par une ponctuation forte :

[10] Un verbe est une action qui indique qu'es que le personnage fait ou comment il est, sa complète le sujet, c'est toujours avant ou après le sujet et un verbe a des terminaison.

5. Les savoirs de l'école, Hachette, 2003 (p. 12) (CE2, CM1, CM2), Les outils de la langue cycle 3. Le nouvel atelier de français, Bordas, 2006 (p. 88), C.L.É.O. Français Manuel d'entraînement CM2, RETZ, 2011 (p. 16), Français, livre unique (collection Caribou) CM2, Istra, 2010 (p. 18), Pépites Français CM2, Magnard, 2013 (p. 122), Interlignes, Étude de la langue - CM2, SED, 2010 (p. 62), Français. Étude de la langue - CM1, CM2, Belin, 2009 (p. 44), L'île aux mots, étude de la langue, cycle 3, Nathan, 2010 (p. 15), Français. Mille-feuille - CM2, Nathan, 2013 (p. 184 et mémo p. 272), À portée de mots, CE2, Hachette, 2009 (p. 18-19). 
Cette complétude est d'ailleurs parfois marquée lexicalement par le mot fin [452] ou l'énoncé Rien d'autre à dire [175].

Les définitions prenant la forme de phrases liées avec ponctuation de phrase restent minoritaires. Elles sont plutôt le fait de réponses courtes comportant une ou deux phrases :

[194] Le verbe est le seul mot qui permet de repérer le temps et qui change de forme.

[253] Le verbe est dans le groupe nominal. Un verbe peut être à l'infinitif ou conjugué.

Dès que les réponses s'allongent, que sont introduits des exemples et des énumérations, la phrase graphique excède généralement la phrase syntaxique. Une difficulté particulière réside en effet dans l'introduction des exemples, ce que font 90 élèves (soit 18\%). Dans les manuels scolaires, le passage du général au particulier s'opère par une phrase non verbale précédée d'une ponctuation forte et comportant le nom exemple suivi de deux points et d'un énoncé en mention marqué typographiquement :

Le verbe permet

- d'exprimer des actions ou de raconter des évènements. Exemple : Ils traquent des animaux redoutables.

- d'indiquer l'état de ce dont on parle. Exemple : L'homo erectus est un remarquable chasseur. (À portée de mots, CE2, Hachette, 2009 (p. 18-19)

Lorsque le mot exemple est employé dans le corpus de CM2, il est rarement accompagné des marques typographiques qui le signalent comme phrase non verbale et l'énoncé en autonymie n'est pas signalé par des guillemets :

[3] Un verbe c'est un verbe qui se conjugue exemple : le verbe savoir, avoir c'est un auxilliaire et être aussi c'est le verbe le plus important.

La sous-utilisation des ressources typographiques nuit également à la lisibilité des énumérations. Là où les manuels procèdent par énumérations binaires avec retrait typographique, les élèves produisent de textes linéaires dans lesquels les alternatives sont éventuellement marquées linguistiquement par quand, soit, ou, mais non soulignées par la ponctuation ou la mise en page. Or, les démarcations graphiques de la définition grammaticale qui ne semblent pas maitrisées ne font pas l'objet d'un enseignement. 


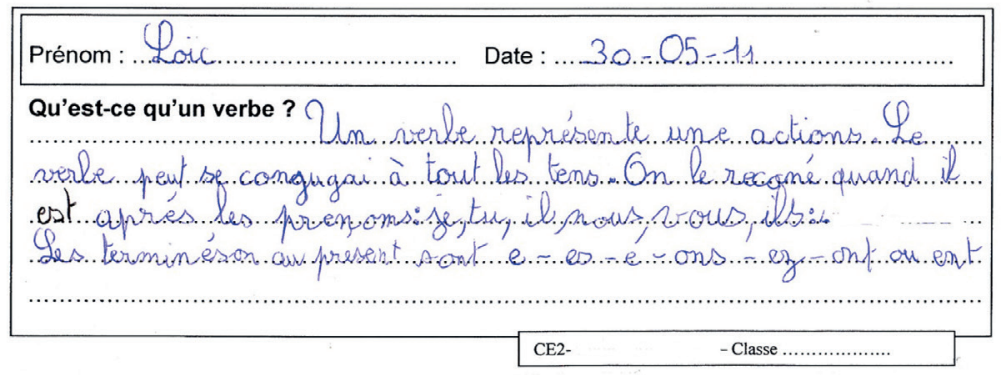

Illustration $n^{\circ} 1$. - Copie scannée d'une réponse d'un élève de CE2 avec une énumération sans retour à la ligne.

À la question «Qu'est-ce qu'un verbe?», les élèves répondent par des énoncés génériques organisés en périodes. Le recul des énoncés non autonomes entre le CE2 et le CM2 montre que le nombre d'élèves capables de produire un discours monogéré augmente. La fréquentation des cahiers de règles et des manuels permet de nombreux apprentissages implicites. Il reste à faire mieux coïncider phrase syntaxique et démarcations graphiques, à gérer les passages du générique au spécifique, avec l'introduction des exemples et la caractérisation des sousclasses de verbes, chose que les manuels ne favorisent pas puisque le verbe est traité en grammaire et les verbes en conjugaison ${ }^{6}$.

\section{La conceptualisation de la relation entre le verbe et la phrase dans les réponses des élèves}

Les réponses écrites des mêmes 491 élèves en début de CE2 puis en fin de CM2 offrent un corpus important pour comprendre la façon dont ils conceptualisent la relation entre le verbe et la phrase. Nous étudierons l'évolution des propriétés linguistiques qui mettent en jeu ces deux notions en prenant appui sur les réponses des mêmes élèves à trois ans d'intervalle. Nous analyserons ensuite les procédures impliquant la phrase auxquelles ont recours les élèves de CM2 pour identifier le verbe et nous nous intéresserons aux propriétés du verbe mettant en jeu la phrase dans leurs définitions. Nous proposerons une typologie des rela-

6. À l'heure où parait cet article, il est trop tôt pour dire si les préconisations des programmes 2016 pour un traitement unifié du verbe seront prises en compte dans les manuels et pourront contribuer à une modification des pratiques. 
tions qu'ils établissent entre ces deux notions, en nous interrogeant sur ce qu'elles permettent d'inférer concernant la compréhension de la notion de prédication, qui n'est pas enseignée en France dans la scolarité obligatoire.

\section{1. Évolution de la place accordée à la phrase dans la description du verbe}

Les réponses des élèves ont été catégorisées à partir d'une grille permettant de coder les propriétés du verbe mentionnées et de les classer selon des entrées sémantiques, syntaxiques, morphologiques, phonologiques, lexicales et textuelles pour pouvoir prendre en compte l'ensemble des propositions. La distribution des entrées retenues par les 491 élèves est la suivante :

\begin{tabular}{|l|c|c|}
\hline \multicolumn{1}{|c|}{491 élèves } & $\begin{array}{c}\text { CE2 } \\
\text { Sept. 2010 }\end{array}$ & $\begin{array}{c}\mathrm{CM} 2 \\
\text { Juin } 2013\end{array}$ \\
\hline $\begin{array}{l}\text { Entrée sémantique } \\
\text { Ex. Un verbe indique l'action. }\end{array}$ & $\mathbf{3 0 \%}$ & $\mathbf{3 9 \%}$ \\
\hline $\begin{array}{l}\text { Entrée morphologique } \\
\text { Ex. Un verbe est un mot que l'on peut } \\
\text { conjuguer. }\end{array}$ & $\mathbf{5 3 \%}$ & $\mathbf{7 8 \%}$ \\
\hline $\begin{array}{l}\text { Entrée syntaxique } \\
\text { Ex. Un verbe est accordé avec le sujet. }\end{array}$ & $\mathbf{2 1 \%}$ & $\mathbf{3 8 \%}$ \\
\hline $\begin{array}{l}\text { Entrée phonologique } \\
\text { Ex. Les verbes du } 2^{2} \text { groupe, on entend issant. }\end{array}$ & $\mathbf{0 , 4 \%}$ & $\mathbf{0 , 2} \%$ \\
\hline $\begin{array}{l}\text { Entrée lexicale } \\
\text { Ex. Le verbe est un mot que l'on trouve dans } \\
\text { le dictionnaire. }\end{array}$ & $\mathbf{0 \%}$ & $\mathbf{1 \%}$ \\
\hline $\begin{array}{l}\text { Entrée textuelle } \\
\text { Ex. C'est grâce à lui que l'histoire avance. }\end{array}$ & $\mathbf{0 \%}$ & $\mathbf{0 \%}$ \\
\hline Entrée non grammaticale & $\mathbf{2 3 \%}$ & $\mathbf{1 , 4 \%}$ \\
\hline
\end{tabular}

Tableau 3. - Répartition des entrées dans les réponses des élèves de $\mathrm{CE}_{2}$ et de $\mathrm{CM}_{2}$. 
Les différentes entrées linguistiques sont en augmentation, ce qui signifie que les réponses complexes associant plusieurs critères sont en augmentation ${ }^{7}$. C'est la conséquence logique d'une structuration grammaticale élaborée par l'école durant ce cycle d'apprentissage. La quasi-disparition des réponses non grammaticales est un indice supplémentaire de cet enseignement de la langue. Cependant, l'entrée morphologique reste dominante et de plus en plus d'élèves l'utilisent pour expliquer le verbe (53\% des élèves de CE2 et $78 \%$ des élèves de CM2). Cette augmentation illustre le poids de la conjugaison dans l'enseignement du verbe à l'école primaire (Gourdet, 2014, p. 225). Les autres distributions confirment les analyses effectuées sur d'autres élèves, comme la quasi-absence de l'entrée phonologique mettant en avant le rapport entre l'oral et l'écrit, et de l'entrée textuelle, absences qui questionnent l'enseignement de ces dimensions à l'école.

Au sein des entrées sémantiques et syntaxiques, notre grille d'analyse spécifie quatre propriétés qui mettent en jeu la phrase : le lien avec le temps de la phrase, le lien avec le sens de la phrase, le positionnement topologique du verbe dans une phrase et la notion de noyau syntaxique ${ }^{8}$. Selon notre grille de lecture et bien que le nombre de propriétés avancées par les élèves soient en augmentation (664 propriétés recensées en début de CE2 et 1185 en fin de CM2), nous constatons une stagnation du poids relatif de ces quatre entrées :

7. C'est pourquoi la somme des pourcentages est nettement supérieure à 100 en CM2.

8. SEM3 : Référence à un mot qui indique le temps du procès, qui permet de se repérer. Explication qui parle d'une partie du discours sémantiquement liée à la notion de temps (il porte la marque du temps sur la phrase); SEM6 : Référence au lien entre le verbe et le sens de la phrase, entrée sémantique dominante (interaction forte avec SYN6); SYN5 : Référence à un positionnement topologique (verbe derrière le sujet par exemple) — La référence peut être en «creux» (une place dans la phrase ou pas de place spécifique dans la phrase...); SYN6 : Le verbe est considéré comme le mot majeur, le noyau de la phrase (son constituant principal/le pivot), le lien direct avec la phrase étant plus ou moins explicite. 


\begin{tabular}{|c|c|c|c|c|}
\hline & \multicolumn{2}{|c|}{ Entrée sémantique } & \multicolumn{2}{|c|}{ Entrée syntaxique } \\
\hline & $\begin{array}{c}\text { Lien avec le } \\
\text { repérage temporel } \\
\text { dans la phrase }\end{array}$ & $\begin{array}{c}\text { Lien avec le sens } \\
\text { de la phrase }\end{array}$ & $\begin{array}{c}\text { Le positionnement } \\
\text { topologique du } \\
\text { verbe dans la } \\
\text { phrase }\end{array}$ & $\begin{array}{c}\text { Le verbe vu } \\
\text { comme le noyau } \\
\text { syntaxique de la } \\
\text { phrase }\end{array}$ \\
\hline $\begin{array}{l}\text { CE2 } \\
\text { Sept. } 2010\end{array}$ & $\begin{array}{c}24 \% \text { des } \\
\text { propriétés } \\
\text { sémantiques * }\end{array}$ & $\begin{array}{c}3 \% \text { des } \\
\text { propriétés } \\
\text { sémantiques }\end{array}$ & $\begin{array}{c}13 \% \text { des } \\
\text { propriétés } \\
\text { syntaxiques }\end{array}$ & $\begin{array}{c}14 \% \text { des } \\
\text { propriétés } \\
\text { syntaxiques }\end{array}$ \\
\hline $\begin{array}{l}\text { CM2 } \\
\text { Juin } 2013\end{array}$ & $\begin{array}{c}24 \% \text { des } \\
\text { propriétés } \\
\text { sémantiques }\end{array}$ & $\begin{array}{c}8 \% \text { des } \\
\text { propriétés } \\
\text { sémantiques }\end{array}$ & $\begin{array}{c}16 \% \text { des } \\
\text { propriétés } \\
\text { syntaxiques }\end{array}$ & $\begin{array}{c}16 \% \text { des } \\
\text { propriétés } \\
\text { syntaxiques }\end{array}$ \\
\hline
\end{tabular}

* Cela signifie qu'en début de CE2, le lien avec le repérage temporel dans la phrase représente $24 \%$ des propriétés sémantiques alors que le lien avec le sens de la phrase ne représente que $3 \%$ de ces mêmes propriétés sémantiques.

Tableau 4. - Répartition des propriétés mettant en jeu la phrase dans les réponses des élèves de $\mathrm{CE}_{2}$ et de $\mathrm{CM}_{2}$.

L'entrée sémantique privilégiée est liée aux variations morphologiques résultant du repérage temporel, tandis que la place et le rôle du verbe dans la phrase restent minorés. De même, l'analyse des occurrences du terme phrase( $s$ ) confirme cette stagnation dans l'importance accordée à la phrase. On dénombre, en début de CE2, 170 occurrences du terme phrase $(s)$ convoqué par 133 élèves (27\% du corpus) et 199 occurrences en CM2 dans les réponses de 137 élèves ( $28 \%$ du corpus).

La très faible évolution des réponses mettant en jeu la phrase incite à penser que le verbe est enseigné et appris à l'école en cloisonnant la morphologie et la syntaxe. Mais derrière cette apparente stagnation quantitative, on constate qu'une partie des élèves de CM2 savent verbaliser leurs moyens d'identifier le verbe et expliquer son rôle dans la phrase.

\subsection{Procédures mettant en jeu le verbe en lien avec la phrase en $\mathrm{CM}_{2}$}

Nous nous sommes intéressés à la façon dont les élèves de CM2 verbalisent le lien avec la phrase quand ils explicitent les moyens d'identification du verbe. À nouveau les dimensions sémantiques et syntaxiques sont sollicitées. Se plaçant en tant que récepteur d'un message, certains décrivent la façon dont s'effectue la prise d'information sur la phrase :

[180] Le verbe; le verbe est un mot qui permet de reconnaître dans l'axe du temps, dans une phrase. 
[144] Le verbe ser a savoir le temps des phrase. Les verbe ser à savoir quand on a fait quelle que chose et qu'on va faire.

Dans les stratégies de compréhension ainsi explicitées, le verbe est reconnu comme un élément nodal.

Adoptant une posture métalinguistique, d'autres élèves décrivent les manipulations qu'ils opèrent sur la phrase pour trouver le verbe. Elles concernent majoritairement la variation temporelle très valorisée au sein des classes, 226 élèves $(46 \%)$ y font référence :

[491] Un verbe sa ce conjugue. Généralement il se trouve au milieu de phrase. On peut le trouver en changant le temps de la phrase et voir quel mot à changer et ses le verbe.

Les élèves convoquent plusieurs procédures, certaines enseignées, d'autres relevant d'apprentissages implicites comme la place médiane du verbe dans la phrase canonique (Riegel et coll., 2009 [1994]).

La variation de personne (61 élèves) et l'encadrement par ne ... pas (8 élèves) sont évoqués de façon plus marginale :

[33] Un verbe est un mots qui sert à donner des informations. Un verbe peut être conjugué ou à l'infinitif. Pour le reconnaître il suffit de changer le temps ou le sujet de la phrase. Il existe plusieurs temps de conjugaison donc le verbe n'est pas toujours écrit de la même façon. Il éxiste des verbes d'état.

[334] Un verbe est un mot qui indique une action. exemple : La poule mange du blé. Le verbe est «mange» car il indique ce que fait la poule. Pour le reconnaître, on peut changer le temps de la phrase. Le mot qui change est forcément le verbe. On peut aussi mettre la négation; ne plus, ne pas, ne jamais. Le mot qui est entre la négation est un verbe.

L'analyse des procédures formulées permet, comme les entretiens métagraphiques, de distinguer les registres sur lesquels les élèves répondent de façon préférentielle, selon qu'ils privilégient une entrée sémantique ou manipulent les énoncés en prenant la langue pour objet.

\subsection{Description des propriétés du verbe mettant en jeu la phrase}

Une analyse des réponses des élèves mettant en jeu le verbe et la phrase permet de faire des hypothèses sur des conceptualisations qui doivent en partie à l'enseignement reçu, mais manifestent aussi des élaborations originales.

Dans leur définition, 30 élèves convoquent la phrase comme le lieu où se trouve le verbe. Cette relation de localisation est présentée dans 
des énoncés dont la généricité varie, selon la modalisation, du simple constat [135] à la propriété définitoire [487] :

[135] Un verbe est un mot qui ce conjugue à plusieurs temps. Il peut il y en avoir dans des phrases. Il peut se conjuguer au temps : Présent Imparfait - futur - p. composé - p. simple - futur antérieur - p. antérieur - plus-que parfait.

[487] Un verbe est un mot qui peut être conjuguer. Il est obligatoirement dans une phrase sauf en cas d'exeption. Il peut être au passé, au présent et au futur. Il doit y avoir un pronom personnel avant le verbe ou un sujet.

Ces définitions présupposent celle d'une phrase canonique dans lequel le verbe est conjugué à une forme personnelle. Ainsi, les variations morphologiques du verbe sont décrites à travers différentes formulations qui mettent en relation le verbe et la phrase :

[61] Un verbe c'est un mot qui est conjugué avec différents temps dans une phrase.

[100] un verbe et un mots qui se conjugue selon le temps de la phrase ou du sujet.

Le verbe changer est employé pour décrire des variations corrélées de verbe et de la phrase :

[82] Cet un mot qui change en fonction de la phrase. exemple : Hier j'ai mangé des nouilles.

Mais le verbe peut également désigner l'agent du changement opérable sur la phrase, formulation que l'on ne rencontre pas dans les manuels à la différence de la précédente, et qui préfigure la fonction prédicative :

[429] C'est un mot qui change le cour de la phrases sa change le temp exemple : Demain je viendrai vous voir.

D'après cet élève, quelle action opère le verbe sur la phrase ? L'expression changer le cours de quelque chose désigne un changement marqué qui affecte le cours de la phrase, c'est-à-dire ce qui est énoncé après le thème, changement qui peut modifier le repérage temporel. En cela, cette formulation préfigure la fonction prédicative. Les variations morphologiques sont donc appréhendées dans un cadre syntaxique. Les énoncés mettant en jeu la phrase, le sujet et le verbe le confirment : 
[92] un verbe est un mot qui peut se conjuguer au present, passée, futur il peut être aussi à l'infinitif. Dans une phrase le verbe est obligatoire dans une phrase il y a un sujet un verbe et un complement

Exemple je mange des frites.

Toutefois, certains élèves dans leur définition ne s'en tiennent pas au prototype de la phrase verbale et s'efforcent de rendre compte des emplois de l'infinitif :

[301] Un verbe est un mots qui peut se conjuguer où être à l'infinitif. Il y a plusieurs temp le passé, le présent, le futur...

[426] un verbe est un verbe conjugues ou à l'infinitif pour respecter une phrase par exemple : passé simple, passé composé etc...

À travers ces formulations, on perçoit le conflit entre la description du verbe que l'on trouve dans la section grammaire des manuels et celle qui figure dans les sections orthographe et conjugaison : en grammaire, le verbe conjugué est présenté comme constituant obligatoire de la phrase verbale, tandis que lorsqu'il s'agit d'orthographier une phrase quelconque ou de classer des formes verbales, la distinction entre formes conjuguées et non conjuguées s'impose.

L'assertion la mieux représentée, dans les définitions du verbe qui mentionnent la phrase, repose sur les propriétés syntaxico-sémantiques du verbe : un mot nécessaire pour faire une phrase. Elle se décline en trois ensembles d'énoncés classés par ordre croissant. Le premier réunit les assertions qui se placent du point de vue de la production - Un verbe ça sert à faire une phrase (5 occurrences). Le second ensemble de réponses présente le caractère indispensable du verbe à travers un raisonnement hypothético-déductif, formulé dans des systèmes hypothétiques - Un verbe est le mot central d'une phrase, s'il n'y a pas de verbe, il n'y a pas de phrase (18 occurrences). Le troisième ensemble d'énoncés, le mieux représenté avec 38 occurrences comporte l'expression donner du sens à :

[145] Un verbe ses ce qui complète une phrase, ca done du sens à la phrase.

Cette expression n'apparait qu'une fois dans le corpus de manuels :

Dans une phrase, l'ordre des mots est très important. Cet ordre permet de lui donner du sens. (Les outils de la langue, Bordas, cycle 3, 2005, p. 16) 
Par-delà le sens de chacun des éléments de la phrase, il y aurait chez certains élèves l'intuition d'une sémantique des relations syntaxiques portée par l'ordre des mots. On peut faire l'hypothèse que ces élèves en saisissent intuitivement la fonction prédicative. En effet, ces assertions ne font pas référence au sens lexical du verbe, mais à la valeur sémantique associée à sa fonction syntaxique à laquelle la grammaire scolaire en France ne donne traditionnellement pas de nom.

[124] Un verbe c'est un mot que l'on conjugue, mais c'est aussi un mot qui donne du sens à la phrase.

Dans plusieurs cas, les élèves précisent ce qu'ils entendent par cette expression. C'est d'abord l'action, propriété sémantique associée à de nombreux verbes, qui est communiquée à l'énoncé entier :

[158] Un verbe est un mot qui donne l'action de la phrases. Il pe s'écrire soit à l'infinitife, en er.

Avec l'action, le verbe devient le moteur du discours :

[73] Un verbe donne une action dans une phrase ou une histoire. Un verbe peut aussi donner des informations suplémentaire, peut aussi donner plus de sens dans les phrases.

Cette intuition de la fonction prédicative du groupe verbal est explicitée dans certains énoncés proposés par ces élèves de $\mathrm{CM} 2$, alors qu'ils sont absents des manuels et qu'il est vraisemblable qu'ils ne figurent pas dans les cahiers de règles de ces mêmes élèves :

[217] Un verbe est la fonction de la phrase. Sa s'accorde toujours avec le pronom personnel ou le sujet.

L'unicité de la fonction prédicative est ici signifiée par le déterminant défini.

Cette même intuition est également portée par un réseau d'images qui convoquent différentes caractéristiques de la fonction prédicative. Le caractère organique de la fonction est décliné à travers plusieurs analogies :

[95] Le verbe est le cœur de la phrase. Une phrase sans verbe n'existe pas à part si c'est un ordre. exemple : Attention à la table ! On peut conjuguer un verbe avec le sujet.

[480] Un verbe est comme le sens d'une phrase. Si il n'y a pas de verbe La phrase na aucun sens. Sait un peu comme un pigeon si il non pas d'aile il ne peut pas voler. 
Les métaphores utilisées montrent qu'ils hésitent entre une analyse logico-sémantique et un modèle de dépendance qui pose le caractère central du verbe :

[483] le verbe et l'action de la phrases y peut se conjuguer ou être a $l^{\prime}$ 'infinitif qui se conjugue avec le sujet il y a 3 groupe $1^{\text {er }}, 2^{\text {ème }}, 3^{\text {ème }} c^{\text {'est }}$ le noyau de la phrase.

À la centralité du verbe correspond l'unicité de la fonction prédicative, que traduisent des énoncés qui pourraient être empruntés aux Éléments de syntaxe structurale de Lucien Tesnière (1959) :

[211] Le verbe c'est comme le conducteur d'une phrase, une phrase n'en n'est pas une sans le verbe, bref le verbe c'est ce qui donne un sens a une phrase.

[20] Un verbe est le chef de la phrase. Il qualifi le sujet. Il se conjugue à toute les personne. Un verbe doit toujours être dans un phrase.

L'examen des réponses a permis d'observer comment les élèves parviennent à définir le verbe dans une tension jamais explicitée (dans leurs réponses comme dans les manuels) entre phrase canonique et phrases réalisées. Par un élargissement du sémantisme d'action, ils accèdent, à la fonction prédicative du verbe, bien qu'elle ne soit pas enseignée. Par la métaphore du noyau, ils saisissent le fonctionnement valenciel, lui aussi occulté.

\section{Conclusion}

À travers l'étude de ce corpus de productions d'élèves, nous avons cherché à cerner les caractéristiques des séquences phrastiques produites et à comprendre comment les élèves conceptualisent la relation entre verbe et phrase. De l'observation de la syntaxe des énoncés produits, on peut conclure que les élèves ont eu peu d'occasion d'observer le fonctionnement de phrases relevant d'un genre pourtant très pratiqué à l'école : la définition d'une notion grammaticale. On peut supposer aussi qu'ils n'ont pas eu l'occasion de comparer d'un point de vue syntaxique des définitions produites à l'oral et à l'écrit. En revanche, la façon dont ils restituent leurs connaissances sur le verbe montre qu'ils ont élaboré un savoir sur la phrase en relation avec le verbe, savoir sur lequel il serait possible de s'appuyer pour concevoir des dispositifs didactiques d'observation de leurs productions. L'introduction, dans les programmes entrant en vigueur en septembre 2016, de la fonction 
prédicat, interdépendante de la fonction sujet, permet d'assigner une fonction au groupe dont le verbe est la tête, fonction dont les écrits d'élèves manifestent l'intuition. L'introduction de cette notion évite que soient confondues une classe grammaticale - le verbe -, , un syntagme - le groupe verbal - et une fonction, qui est une relation entre syntagmes interdépendants. Elle permet ainsi de clarifier les niveaux d'analyse et d'articuler la construction lexicale d'un verbe donné, avec ses arguments, à celle de la phrase dans laquelle il assume la fonction prédicat en procédant par paliers de traitement (Combettes, 2005; Elalouf, 2014). Cette notion, déjà présente dans les programmes suisses romands (Bronckart, 2001) et québecois (Chartrand et. coll., 2011 [1999]) devrait permettre de poser un métaterme sur un fonctionnement linguistique que plusieurs réponses d'élèves tentent de cerner; elle devrait aussi aider à la conceptualisation de la phrase syntaxique en production en cernant ce qui change le cours de la phrase.

\section{RÉFÉRENCES BIBLIOGRAPHIQUES}

Bally, Charles. (1909). Traité de stylistique française. Paris : Klincksieck. Berrendonner, Alain. (2002). Les deux syntaxes. Dans M. Charolles, P. Le Goffic \& M.-A. Morel (dir.), Y a-t-il une syntaxe au-delà de la phrase?, Verbum, 24(1-2), 23-35.

Boré, Catherine \& Boresdon, Catherine. (2013). La phrase selon les brouillons : un trajet entre l'oral et l'écrit. Le français aujourd'hui, $181,13-24$.

BRONCKART, Jean-Paul. (2001). Enseigner la grammaire dans le cadre de l'enseignement rénové de la langue. Genève : DIP (Cahier du secteur des langues, 75).

Chartrand, Suzanne, Aubin, Denis, Blain, Raymond \& Simard, Claude. (2011 [1999]). Grammaire pédagogique du français d'aujourd'hui. Montréal : Chenelière Éducation.

Combettes, Bernard. (2005). Pour une rénovation des contenus en grammaire de phrase : l'apport des approches fonctionnelles. Pratiques, 125 $126,7-24$.

Elalouf, Marie-Laure. (2014). La notion de phrase de base dans la pratique des enseignants français : choix terminologiques et enjeux théoriques. Repères, 49, 33-55.

FISHER, Carole. (2004). La place des représentations des apprenants en didactique de la grammaire. Dans C. Vargas (dir.), Langue et études de la langue. Approches linguistiques et didactiques (p.383-393). Aix-enProvence : Publications de l'Université de Provence. 
Gourdet, Patrice. (2013). Le «cahier de règles » à l'école élémentaire, outil institutionnel et référence grammaticale : le cas du verbe en CE2. Dans O. Bertrand \& I. Schaffner (dir.), Enseigner la grammaire (p. 265-285). Palaiseau : Éditions de l'École polytechnique.

GouRdET, Patrice. (2014). Les explications linguistiques sur le verbe. Un suivi sur une année scolaire d'une cohorte de CE2. Dans M.-N. Roubaud \& J.-P. Sautot (dir.), Le verbe en friche. Approches linguistiques et didactiques (p. 217-234). Bruxelles : Peter Lang.

GouRdet, Patrice \& Cogis, Danièle. (2014). Un verbe c'est quelque chose : emploi «profane» ou emploi «savant» du métalangage à l'école élémentaire? Dans M. Lecolle (dir.), Métalangage et expression du sentiment linguistique "profane», Le discours et la langue, 6(1), 47-62.

Paolacci, Véronique \& Rossi-Gensane, Nathalie. (2012). Quelles images de la phrase dans les écrits d'élèves de fin d'école primaire française? Description linguistique et réponses didactiques aux difficultés des élèves. Actes $d u 3^{\mathrm{e}}$ Congrès mondial de linguistique française (vol. 1, p. 341-359). EDP Sciences. <http://dx.doi.org/10.1051/shsconf/201201 00143>.

Riegel, Martin, Pellat, Jean-Christophe \& Rioul, René. (2009 [1994]). Grammaire méthodique du français. Paris : PUF.

TeSNIÈRE, Lucien. (1959). Éléments de syntaxe structurale. Klincksieck. Tomassone, Roberte. (2002). Pour enseigner la grammaire. Delagrave. 